\title{
Words Medicine Residents Use to Describe Substance Use Disorders After Aftending a Mutual Support Group Meeting
}

J Gen Intern Med 36(12):3913-4

DOI: $10.1007 / \mathrm{s} 11606-020-06507-5$

(C) Society of General Internal Medicine 2021

\section{INTRODUCTION}

The medical community has increasingly recognized the benefits of non-stigmatizing and person-first language (i.e., putting the person before the diagnosis and describing what a person has, not who a person is) in the treatment of persons with substance use disorders (SUDs). Though guidelines on person-first language exist, ${ }^{1,2}$ it is unknown what actual words physicians use when describing SUDs and persons with SUDs in practice. Internal medicine residents are a key group of individuals to study, given their frequent interactions with patients with SUD in inpatient and outpatient settings during their training. The aim of this study was to identify what terms medical residents use to describe SUDs and people with SUDs in a written reflection immediately after attending a mutual support group (MSG) meeting.

\section{METHODS}

As part of a previously described educational intervention, ${ }^{3} 63$ internal medicine residents from all 3 post-graduate years from the University of Pittsburgh Medical Center were required to attend a MSG meeting, either narcotics anonymous (NA) or alcoholics anonymous (AA), during the 2017-2018 academic year. After attending the MSG meeting, residents were asked to write a half-page reflection on their experience using the prompt: "Reflect on your experience at the meeting". Residents received no formal training on person-first and/or nonstigmatizing language for SUDs at the time of this study.

A secondary analysis of the written reflections was performed using qualitative content analysis. ${ }^{4}$ We coded the frequency of words residents used in their written reflections to describe SUDs and characteristics of MSG attendees/ persons with SUD. The SUD codes were further refined by the context of what the residents described, resulting in the following categories: (1) general words used to describe SUDs, (2) words used to describe opioid use disorder (OUD), and (3) words used to describe alcohol use disorder

Received September 1, 2020

Accepted December 17, 2020

Published online January 6, 2021
(AUD). The primary investigator (A.K.) read all reflections and met with co-investigators (K.L. and G.F.) to develop a preliminary coding scheme. Two authors (K.L. and G.F.) coded all reflections independently and A.K. and A.C. met to compare their coding and adjudicate all differences. Coding was performed utilizing the ATLAS.ti software (version 6).

\section{RESULTS}

Of the 68 residents who received the previously described educational intervention, 63 (93\%) completed a written reflection. Within the written reflections, the most frequently coded words to describe SUDs in general were addiction (coded 84 times), problem (31 times), and disease (27 times) (Table 1). Alcohol use disorder was described in 5 different ways including alcoholism (coded 32 times), alcohol abuse ( 9 times), and alcohol use disorder (6 times). Similarly, OUD was described in 6 different ways including narcotic abuse (coded 2 times), opiate abuse ( 2 times), and opioid use disorder (1 time). The most common adjectives that residents used to describe MSG attendees were sober (coded 33 times), alcoholic (25 times), addict(s) (17 times), and clean (17 times) (Table 2).

Table 1 Coded Words Residents Used to Describe Substance Use Disorders in the 63 Written Reflections

\begin{tabular}{ll}
\hline Coded word & Frequency, $\boldsymbol{N}$ \\
\hline General substance use disorder & \\
Addiction(s) & 84 \\
Problem(s) & 31 \\
Disease(s) & 27 \\
Substance abuse & 8 \\
Substance use disorder & 8 \\
Drug use & 6 \\
Substance user(s) & 4 \\
Drug abuse & 2 \\
Substance abuse disorder & 1 \\
Substance issues & 1 \\
Substances of abuse & 1 \\
Drug addiction & 1 \\
Alcohol use disorder & \\
Alcoholism & 32 \\
Alcohol abuse & 9 \\
Alcohol use disorder & 6 \\
Alcohol dependence & 5 \\
Alcohol addiction & 3 \\
Opioid use disorder & \\
Narcotic abuse & 2 \\
Opiate abuse & 2 \\
Narcotic abuser(s) & 1 \\
Narcotic addiction & 1 \\
Opioid addiction & 1 \\
Opioid use disorder & 1 \\
\hline
\end{tabular}


Table 2 Adjectives Used to Describe MSG Meeting Attendees in the 63 Resident Written Reflections

\begin{tabular}{ll}
\hline \hline Coded word & Frequency, $\boldsymbol{N}$ \\
\hline Sober & 33 \\
Alcoholic & 25 \\
Addict(s) & 17 \\
Clean & 17 \\
Powerful & 11 \\
Overcome(ing) & 8 \\
Fight(ing) & 5 \\
Powerless & 5 \\
Quitting & 5 \\
Abstinent & 4 \\
Recovering & 4 \\
Suffering & 4 \\
Drunk/a drunk & 3 \\
Excited & 3 \\
Abusing & 2 \\
Afflicted & 2 \\
Lonely & 2 \\
Recovering alcoholic & 2 \\
Solemn & 2 \\
\hline
\end{tabular}

\section{CONCLUSIONS}

Qualitative content analysis demonstrated residents' use of a variety of words to describe SUDs and MSG attendees after attending an MSG meeting. In general, residents used outdated language such as "addict" and "alcoholic" rather than nonstigmatizing, person-first language to describe MSG attendees in their written reflections. These results are consistent with prior research on medical students, ${ }^{5}$ suggesting that despite recommendations to use person-first language, outdated terminology for SUD continues to be a widespread problem.

We previously reported that after attending MSG meetings, residents had more positive attitudes towards people with SUD and had improved perspective taking. ${ }^{3}$ Thus, our findings may suggest that residents were reflecting the language they heard at a MSG meeting where the culture is often for participants refer to themselves as an "alcoholic" or "addict."

Our study was limited by a small sample size of one medical specialty at a single academic institution, and our results may not be generalizable to trainees in other specialties and institutions. We analyzed reflective essays and thus captured only written, not spoken language.

In conclusion, residents used a variety of words, many of which were not person-first terms, to describe SUDs and MSG attendees after attending an MSG meeting. These results suggest that while sending trainees to MSGs is beneficial, ${ }^{3,6}$ language used at these meetings is not clinical language; therefore, future educational interventions that involve MSGs should be complemented by explicit instruction in current SUD terminology.
Acknowledgments: We wish to thank Ethan Lennox, MA, Division of General Internal Medicine, University of Pittsburgh School of Medicine, for his help with manuscript editing and the residents who participated in this study for sharing their experiences so generously.

Amy J. Kennedy, MD, MS ${ }^{1}$

Keely Lombardi ${ }^{2}$

Gretchen Fruehstorfer ${ }^{2}$

Megan Hamm, $\mathrm{PhD}{ }^{3}$

Melissa McNeil, $M D, M P H^{3}$

Andrea Carter, $M D, M S^{3}$

${ }^{1}$ Ambulatory Care Network, Los Angeles County Department of Health Services, 1670 E 120th Street, Los Angeles, CA 90059, USA

${ }^{2}$ University of Pittsburgh, 4200 Fifth Ave, Pittsburgh, PA 15260, USA

${ }^{3}$ Division of General Internal Medicine, University of Pittsburgh School of Medicine, Pittsburgh, PA, USA

Corresponding Author: Andrea Carter, MD, MS; Division of General Internal Medicine, University of Pittsburgh School of Medicine, Pittsburgh, PA, USA (e-mail: carterae@upmc.edu).

Funding The primary investigator was supported by an HRSA T32 (T32HP22240) training grant for a portion of the data analysis and writing of the manuscript.

\section{Compliance with Ethical Standards:}

Conflict of Interest: The authors declare that they do not have a conflict of interest.

Ethical Approval: This study was given an exempt approval (PRO17080500) by the University of Pittsburgh Institutional Review Board (IRB).

\section{REFERENCES}

1. Broyles LM, Binswanger IA, Jenkins JA, et al. Confronting inadvertent stigma and pejorative language in addiction scholarship: A recognition and response. Subst Abus. 2014;35(3):217-221.

2. Botticelli MP, Koh HK. Changing the language of addiction. JAMA - J Am Med Assoc. 2016;316(13):1361-1362.

3. Kennedy AJ, McNeil M, Hamm M, Abril Cameron FA, Carter AE. Internal Medicine Resident Perceptions of Patients with Substance Use Disorder After Attending a Mutual Support Group Meeting. J Gen Intern Med. 2020;35(3):918-921.

4. Elo S, Kyngäs H. The qualitative content analysis process. $J$ Adv Nurs. 2008;62(1):107-115.

5. Camp ME, Cole AG, Sadler JZ. Addicts and Admits: Metonymy in Medical Students' Reflective Writing. Teach Learn Med. 2020;32(1); 23-33.

6. Grogan $\mathbf{K}$, Zerbo E. Breaking down the wall: A narrative approach to addiction. Med Humanit. 2015;41: 143-144.

Publisher's Note: Springer Nature remains neutral with regard to jurisdictional claims in published maps and institutional affiliations. 